\title{
Perturbation about the Mean Field Critical Point
}

\author{
Jean Bricmont ${ }^{\dagger \star 1}$ and Jean-Raymond Fontaine ${ }^{\star}{ }^{2}$ \\ 1 Department of Mathematics, Princeton University, Princeton, NJ 08544, USA \\ ${ }^{2}$ Department of Mathematics, Rutgers University, New Brunswick, NJ 08903, USA
}

Appendix

\section{Eugene Speer}

Department of Mathematics, Rutgers University, New Brunswick, NJ 08903, USA

\begin{abstract}
We consider two models that are small perturbations of Gaussian or mean field models : the first one is a double well $\lambda / 4 \phi^{4}-\sigma / 2 \phi^{2}$ perturbation of a massless Gaussian lattice field in the weak coupling limit $(\lambda \rightarrow 0, \sigma$ proportional to $\lambda$ ). The other consists of a spin $1 / 2$ Ising model with long-range Kac type interactions; the inverse range of the interaction, $\gamma$, is the small parameter. The second model is related to the first one via a sine-Gordon transformation. The lattice $\mathbb{Z}^{d}$ has dimension $d \geqq 3$.

In both cases we derive an asymptotic estimate to first order (in $\lambda$ or $\gamma^{2}$ ) on the location of the critical point. Moreover, we prove bounds on the remainder of an expansion in $\lambda$ or $\gamma$ around the Gaussian or mean field critical points.

The appendix, due to E. Speer, contains an extension of Weinberg's theorem on the divergence of Feynman graphs which is used in the proofs.
\end{abstract}

\section{Introduction}

It is well known that rigorous and accurate estimates on the location of the critical point of statistical mechanical systems are in general very difficult to obtain. This is related to the fact that high and low temperature expansions are not known to converge up to the critical temperature. However, for models that are close to Gaussian or mean-field theories, we can obtain more detailed information.

Specifically, we shall consider three cases. The nearest-neighbour Ising model on $\mathbb{Z}^{d}$ in the limit of $d \rightarrow \infty$; a lattice field theory in the weak coupling limit, and an Ising model with weak long range interaction of Kac type. For the nearest neighbour Ising model it is straightforward to show, combining the infrared bounds [1] and Fisher's mean field bounds on $T_{c}$ [2] that

$$
T_{c}(d) / 2 d=1-1 / 2 d+o(1 / d)
$$

* Present address: Institut de Physique Theorique, Université de Louvain, Belgium 
(with n.n. coupling $J=1$ ) (This observation was also made by B. Simon [3].) In the two other cases we obtain a similar first order correction on the critical temperature using results from [2,4-6] but we can also control an expansion about the Gaussian or mean field limit for the correlation functions.

The lattice field theory is defined as follows: let for each $x \in \mathbb{Z}^{d}, \phi_{x} \in \mathbb{R}$ and let the Hamiltonian be

$$
H=1 / 2 \sum_{\langle x y\rangle}\left(\phi_{x}-\phi_{y}\right)^{2}+\lambda / 4 \sum_{x} \phi_{x}^{4}-\sigma / 2 \sum_{x} \phi_{x}^{2} .
$$

We set $\beta=T^{-1}=1$ since it can be absorbed in $\lambda, \sigma$ by a change of scale on $\phi$. The point $\lambda=\sigma=0$ is the Gaussian massless (critical) theory. There exists a curve $\sigma_{c}(\lambda)$ of critical points starting from that point. We compute the tangent of that curve at the origin:

$$
\sigma_{c}(\lambda)=3 \lambda C_{00}+o(\lambda)
$$

where

$$
C_{00}=(-\Delta)^{-1}(0,0)=(2 \pi)^{-d} \int_{-\pi}^{\pi}\left[2 \sum_{\alpha=1}^{d}\left(1-\cos k_{\alpha}\right)\right]^{-1} d^{d} k .
$$

This holds for any $d \geqq 3$.

An analogous formula holds for any even polynomial with positive coefficients instead of $\phi^{4}$.

In order to express our second result, fix $\sigma / \lambda<3 C_{00}-\varepsilon$. Then, for any $n$, we can write the free energy and the correlation functions of the system as a sum of Gaussian integrals depending on $\lambda$ and multiplied by powers of $\lambda$ plus a remainder of order $\lambda^{n}$.

We describe now the second model, namely the Ising model with Kac-type interactions.

In order to study the liquid gas transition, Kac [7] introduced a model of classical statistical mechanics where the two body potential is of the form:

$$
u_{\gamma}(r)=q(r)+\gamma^{d} w(\gamma r) .
$$

Here $q(r)$ is a short range repulsive interaction and $w(r)$ is an attractive long-range interaction, e.g. $w(r)=-\exp (-r), d$ is the dimension of the space, and for all $\gamma$,

$$
\int_{\mathbb{R}^{d}} \gamma^{d} w(\gamma r) d^{d} r=\int w(r) d^{d} r
$$

is independent of $\gamma$. As $\gamma \rightarrow 0$ the interaction $\gamma^{d} w(\gamma r)$ becomes at the same time weaker and more long-ranged, Baker [8] observed that this limit gives rise to a transition of the mean field type. Moreover it was shown by Kac, Uhlenbeck and Hemmer [9] for $d=1$ and then in general by Lebowitz and Penrose [10] that the free energy of these models converges as $\gamma \rightarrow 0$ to the van der Waals free energy together with the Maxwell or double-tangent construction.

Given this fact, it is of interest to try to obtain some information on the models with $\gamma$ small but nonzero by perturbing around mean field theory. The inverse range of the interaction, $\gamma$, is used as a small parameter. Systematic expansions of this type were first introduced by Brout [11] and studied by many authors [1215] (see e.g. [16] for a review). 
We study a lattice version of these models where $q(r)$ is replaced by a hard-core condition : one particle at each site of the lattice. These lattice gases with attractive potentials can be transformed into Ising models with ferromagnetic interactions where the Hamiltonian is

$$
-H=\sum_{(x, y)} J_{x y}(\gamma) \sigma_{x} \sigma_{y}+h_{\gamma} \sum_{x} \sigma_{x}
$$

(to have phase transitions we will set $h_{\gamma}=0$ ). Several models of this type were considered by Kac and Helfand [17].

We study a model very similar to model $c$ of [17], namely we choose

$$
J_{x y}(\gamma)=\gamma^{2}\left(-\Delta+\gamma^{2}\right)^{-1}(x, y),
$$

where $\Delta$ denote the finite difference Laplacian on $\mathbb{Z}^{d}$. Notice that $J_{x y}(\gamma)$ is positive (ferromagnetic), translation invariant, decays exponentially at a range $\gamma$ in all directions, and that

$$
\sum_{y \in \mathbb{Z}^{d}} J_{0 y}(\gamma)=1
$$

Therefore the mean field critical temperature is equal to 1. For similar models, $T_{c}(\gamma)$ has been computed perturbatively in two and three dimensions to first order $[18,19]$. We prove here that

$$
T_{c}(\gamma)=1-C_{00} \gamma^{2}+o\left(\gamma^{2}\right)
$$

This is analogous to (2) but the proof is much easier (and similar to the $d \rightarrow \infty$ case). Our main result about this model concerns the $\gamma$ expansion: fix $(\beta-1) / \gamma^{2}=C_{00}-\varepsilon$ (in particular we can take $\beta=1=\beta_{c}$ (mean field)). Then, as in the $\phi^{4}$ case, we can express, for each $n$, the free energy and the correlation functions as a sum of Gaussian integrals depending on $\gamma$ and show that the remainder is of order $\gamma^{n}$. This is quite similar to the other $\gamma$ expansions studied in the literature (see [16]), the only new result being rigorous bounds on the remainder. Such bounds were obtained previously by Siegert, for the free energy, to first order in $\gamma[15]$ (where our $\gamma$ corresponds to $\gamma^{1 / d}$ ).

\section{The Results}

\section{A. The Models}

1. The Ising Model. At each point of a lattice $\mathbb{Z}^{d} d \geqq 3$ there is a spin variable $\sigma_{x}= \pm 1$ and for each $\gamma>0$ we consider the Hamiltonian given, in a finite volume $\Lambda$, by

$$
-H_{\Lambda}=\sum_{(x, y) \subset \Lambda} J_{x y}(\gamma) \sigma_{x} \sigma_{y}
$$

where each pair $(x, y)$ is counted once and where either

a)

$$
\begin{gathered}
J_{x y}=1 \text { if }|x-y|=1, \\
0 \text { otherwise, }
\end{gathered}
$$


or

b)

$$
J_{x y}=\gamma^{2}\left(-\Delta+\gamma^{2}\right)^{-1}(x, y)
$$

with

$$
\begin{aligned}
(-\Delta)(x, y) & =2 d & \text { if } & x=y, \\
& =-1 & \text { if } & |x-y|=1 .
\end{aligned}
$$

We have put free boundary conditions on $\Lambda$. We shall use the notation $\left(-\Delta+\gamma^{2}\right)^{-1}(x, y)=C_{x y}^{\gamma}$ which is explicitly given by

$$
C_{x y}^{\gamma}=(2 \pi)^{-d} \int_{-\pi}^{\pi} \frac{\exp (i \vec{k}(x-y))}{2 \sum_{\alpha=1}^{d}\left(1-\cos k_{\alpha}\right)+\gamma^{2}} d^{d} k .
$$

We write $C_{x y}$ for the case $\gamma=0$. The Gibbs measure $\mu_{\Lambda, \beta}$ on $\{-1,+1\}^{\Lambda}$ at inverse temperature $\beta=T^{-1}$ is given by

$$
\begin{aligned}
\mu_{\Lambda, \beta}\left(\left\{\sigma_{x}\right\}\right) & =Z_{\Lambda, \beta}^{-1} \exp \left(-\beta H_{\Lambda}\left(\left\{\sigma_{x}\right\}\right)\right) \\
Z_{\Lambda, \beta} & =\sum_{\substack{\sigma_{x}= \pm 1 \\
x \in \Lambda}} \exp \left(-\beta H_{\Lambda}\left(\left\{\sigma_{x}\right\}\right)\right) .
\end{aligned}
$$

As $\Lambda \uparrow \mathbb{Z}^{d}$, the correlation functions $\int \prod_{x \in A} \sigma_{x} d \mu_{\Lambda, \beta}(A \subset \Lambda)$ converge, by Griffiths inequalities [20], and define a Gibbs state $\langle>(\beta)$. As $\beta$ varies both models undergo a phase transition which can be characterized as follows:

a) For $\beta$ small $\left\langle\sigma_{0} \sigma_{x}\right\rangle \leqq \exp (-m|x|)$ for some $m>0$. We say that there is exponential clustering (actually, by $\mathrm{FKG}$ inequalities all truncated correlation functions decay exponentially).

b) For $\beta$ large, $\left\langle\sigma_{0} \sigma_{x}\right\rangle \nrightarrow 0$ as $|x| \rightarrow \infty$. We say that there is long-range (LRO). By Griffiths inequalities [21], this implies a spontaneous magnetization.

2. The $\phi^{4}$ Models. At each point $x \in \mathbb{Z}^{d}$ there is a real variable $\phi \in \mathbb{R}$ and we define the finite volume Gibbs measure on $\mathbb{R}^{|\lambda|}$ by

$$
d \mu_{\Lambda, h} \sim Z_{\Lambda}^{-1} \exp \left[(1 / 2)\left(\phi, \Delta_{D} \phi\right)-(\lambda / 4) \sum_{x \in \Lambda} \phi_{x}^{4}+(\sigma / 2) \sum_{x \in \Lambda} \phi_{x}^{2}+\mathrm{h} \sum_{x \in \Lambda} \phi_{x}\right] \prod_{x \in \Lambda} \mathrm{d} \phi_{x}
$$

where $\Delta_{D}$ is the lattice Laplacian with Dirichlet boundary conditions on $\Lambda$, $\phi=\left(\phi_{x}\right)_{x \in \Lambda}$ and we take $\lambda>0, \sigma$ real. $Z_{\Lambda}$ is the normalization factor. We define our infinite volume state $\langle>$ by:

$$
\left\langle\prod_{x} \phi_{x}^{n_{x}}\right\rangle=\lim _{h \downarrow 0} \lim _{\Lambda \uparrow \mathbb{Z}^{d} \mathbb{R} \backslash \Lambda \uparrow} \int_{x} \prod_{x} \phi_{x}^{n_{x}} d \mu_{\Lambda, h}
$$

The limits exist by monotonicity [20] and superstability estimates [23]. For this model, as in the Ising model, there is a phase transition as $\sigma$ is varied, for fixed $\lambda$ : for $\sigma$ negative there is exponential clustering and for $\sigma$ large there is long-range order (this can be shown using a Peierls argument as in [24]). 
The relation between model $1 \mathrm{~b}$ and model 2 is provided by:

3. The Sine-Gordon Transformation. This is based on the following identity introduced by Kac [7]:

$$
\exp \left(\frac{\beta \gamma^{2}}{2} \sum_{x, y \in \Lambda} C_{x y} \sigma_{x} \sigma_{y}\right)=\left\langle\exp \gamma \sqrt{\beta} \sum_{x \in A} \sigma_{x} \phi_{x}\right\rangle,
$$

where $\phi_{x}$ is a set of Gaussian random variables with covariance $\left\langle\phi_{x} \phi_{y}\right\rangle=C_{x y}$. This formula holds whenever the matrix $C_{x y}$ is positive definite. We take here $C_{x y}=C_{x y}^{\gamma}$. Summing over $\sigma_{x}$ in (9) we obtain

$$
\begin{gathered}
Z_{\Lambda, \beta, \gamma}=\left\langle\prod_{x \in \Lambda} \cosh \left(\sqrt{\beta} \gamma \phi_{x}\right)\right\rangle_{\gamma}, \\
\left\langle\sigma_{x} \sigma_{y}\right\rangle(\beta, \gamma)=\left\langle\tanh \left(\sqrt{\beta} \gamma \phi_{x}\right) \tanh \left(\sqrt{\beta} \gamma \phi_{y}\right)\right\rangle_{\Lambda} \text { for } x \neq y,
\end{gathered}
$$

where \langle\rangle is the Gaussian measure of covariance $C_{x y}^{\gamma}$ and \langle\rangle$_{A}$ in the right hand side of (11) is given by

$$
\langle\cdot\rangle_{\Lambda}=\frac{\left\langle\cdot \prod_{x \in \Lambda} \cosh \left(\sqrt{\beta} \gamma \phi_{x}\right)\right\rangle_{\gamma}}{\left\langle\prod_{x \in A} \cosh \left(\sqrt{\beta} \gamma \phi_{x}\right)\right\rangle_{\gamma}}
$$

In this way our long range Ising model has been transformed into a lattice field theory with a Gaussian measure of mass $\gamma$ and a perturbation $V\left(\phi_{x}\right)=\ln \cos \sqrt{\beta} \gamma \phi_{x}$. We define

$$
\left\langle\prod_{x} \phi_{x}^{n_{x}}\right\rangle=\lim _{\Lambda \uparrow \mathbb{Z}^{d}}\left\langle\prod_{x} \phi_{x}^{n_{x}}\right\rangle_{\Lambda} n_{x} \geqq 0
$$

The limit exists by monotonicity [20] and superstability estimates [23]. Note that, if we expand

$$
\log \cosh \sqrt{\beta} \gamma \phi \simeq \frac{1}{2} \gamma^{2} \beta \phi^{2}-\frac{1}{2} \gamma^{4} \beta^{2} \phi^{4}
$$

and identify

$$
\lambda=\gamma^{4} \beta^{2} / 3, \quad \sigma=(\beta-1) \gamma^{2}
$$

we get the Hamiltonian (8).

\section{B. Estimates on the Critical Temperature}

We give our estimates on the critical temperature of the three models described above. In model $(a)$ we vary the lattice dimension $d$. In the two other models $d$ is fixed $(d \geqq 3)$ and we vary either $\gamma$ or $\lambda$. We shall generally set $d=3$ for these models.

Theorem 1. 1) For model 1a) let $T / 2 d=1-b / 2 d$
a) if $b<1$ there is exponential clustering for all $d$,
b) if $b>1$, for d large enough, there is long range order.
2) For model $1 b)$, let $\beta=1+b \gamma^{2}$
a) if $b<C_{00}$ for $\gamma$ small enough, there is exponential clustering,
b) if $b>C_{00}$ there is long range order for all $\gamma$. 
Theorem 2. For model 2, let $\sigma=b \lambda$

a) if $b<3 C_{00}$ for $\lambda$ small enough there is exponential clustering,

b) if $b>3 C_{00}$ there is long range order for all $\lambda$.

Remarks. 1) We may summarize the two theorems as follows:

$$
\begin{aligned}
T_{c}(d) / 2 d & \simeq 1-1 / 2 d, \\
T_{c}(\gamma) & \simeq 1-C_{00} \gamma^{2}, \\
\sigma_{c}(\lambda) & \simeq 3 \lambda C_{00} .
\end{aligned}
$$

2) One can extend this theorem to the case where we replace

$$
\frac{\lambda \phi^{4}}{4}-\frac{\sigma \phi^{2}}{2} \text { by } \lambda \sum_{i=2}^{n} a_{i} \phi^{2 i}-\frac{\sigma \phi^{2}}{2}
$$

with $a_{i} \geqq 0$. Then $\sigma_{c}(\lambda)$ is given to first order in $\lambda$ by:

$$
\sigma_{c}(\lambda)=2 \lambda \sum_{i=2}^{n} \frac{(2 i) ! a_{i}}{(i-1) ! 2^{i}}\left(C_{00}\right)^{(i-1)}
$$

(see Remark at the end of Sect. IV).

\section{Perturbation Theory}

Let us start with the $\lambda \phi^{4}$ model. A crucial role is played by the Wick ordering formula :

$$
: \phi^{4}:_{m}=\phi^{4}=6 C_{00}^{m} \phi^{2}+3\left(C_{00}^{m}\right)^{2}
$$

(the constant is irrelevant in what follows).

If $\sigma<3 \lambda C_{00}$, then there exists a $m>0$ such that

$$
\frac{\lambda \phi^{4}}{4}-\frac{\sigma \phi^{2}}{2}=\frac{\lambda: \phi^{4}: m}{4}+\frac{m^{2} \phi^{2}}{2}+C .
$$

Here $m$ is the solution of

$$
m^{2}=3 \lambda C_{00}^{m}-\sigma,
$$

and if $\sigma=\left(3 C_{00}-\varepsilon\right) \lambda$ then $m^{2}$ is of order $\varepsilon \lambda$ as $\lambda \rightarrow 0$.

Theorem 3. Let $\sigma=\left(3 C_{00}-\varepsilon\right) \lambda$ and let $A$ be a finite subset of $\mathbb{Z}^{d}$; then for any $\varepsilon>0$ and any $l \in \mathbb{N}$

$$
\left\langle\prod_{x \in A} \phi_{x}^{n_{x}}\right\rangle=\sum_{k=0}^{l} G_{k}(\lambda)+R_{l}(\lambda),
$$

where $\lim _{\lambda \rightarrow 0} R_{l}(\lambda) \lambda^{-l}=0,\left\langle>\right.$ is the state defined in Sect. II. A, $n_{x} \in N$ and $G_{k}(\lambda)$ is the sum of all Feynman graphs of order $k$ in $\lambda$ for the lattice $\lambda: \phi^{4}:_{m} / 4$ theory with $n_{x}$ external legs at each $x \in \mathbb{Z}^{d}$; these graphs are computed in a Gaussian field of mass $m$ where $m$ is given by (16). 
Remarks. 1) One can also expand the free energy, by differentiating it with respect to $\lambda$ and expanding the resulting expectation values.

2) If we take $\left\langle\phi_{0}^{2}\right\rangle$ then the first term in (17) is $C_{00}^{m(\lambda)}$ (with an error of order $\lambda$ ). Since $C_{00}^{m(\lambda)} \simeq C_{00}-0(m(\lambda))$ and $m(\lambda)$ is order $\sqrt{\lambda},(17)$ does not give rise to an asymptotic expansion in powers of $\lambda$, because of the $\lambda$ dependence of $m$. For higher orders even logarithmic terms may appear. Nevertheless, this expansion expresses all the information up to order $\lambda^{l}$ in terms of Gaussian integrals.

2. Let us turn now to the Ising model and the $\gamma$ expansion. Due to the SineGordon transformation [formula (11) and generalizations of it to general correlation functions], we need only consider an expansion for the $\log \cosh$ theory. First we write:

$$
-1 / 2 \gamma^{2} \phi^{2}+\log \cosh \sqrt{\beta} \gamma \phi=\frac{(\beta-1)}{2} \gamma^{2} \phi^{2}+\sum_{i=2}^{n} b_{i} \beta^{i} \gamma^{2 i} \phi^{2 i}+\text { Remainder },
$$

where $b_{i}$ are the coefficients of the Taylor expansion around zero. Then we Wick order:

$$
-1 / 2 \gamma^{2} \phi^{2}+\log \cosh \sqrt{\beta} \gamma \phi=-\frac{m^{2} \phi^{2}}{2}+\sum_{i=2}^{n}(-1)^{i+1} a_{2 i} \beta^{i} \gamma^{2 i}: \phi^{2 i}:_{m}+\text { Remainder }
$$

(without changing the remainder). Since the first term $(i=2)$ is of order $\beta^{2} \gamma^{4} / 12$ we see that this Wick ordering is possible (with $m>0$ ) for $\gamma$ small as soon as $\beta-1$ $<C_{00} \gamma^{2}$ and that if $\beta-1=\left(C_{00}-\varepsilon\right) \gamma^{2}, m^{2}$ is of order $\varepsilon \gamma^{4}$ as $\gamma \rightarrow 0$. Note that adding a term of order $2 n$ to the polynomial changes $m^{2}$ in (19) but only by an amount of order $\gamma^{2 n}$. Now we can state

Theorem 4. Let $\beta=1+\left(C_{00}-\varepsilon\right) \gamma^{2}$ and let $A$ be a finite subset of $\mathbb{Z}^{d}$; then for any $\varepsilon>0$ and any $l \in \mathbb{N}$.

$$
\left\langle\prod_{x \in A} \phi_{x}^{n_{x}}\right\rangle=\sum_{k=0}^{l} G_{2 k}(\gamma)+R_{2 l}(\gamma)
$$

where $\lim _{\gamma \rightarrow 0} R_{2 l}(\gamma) \gamma^{-2 l}=0 ;\langle\rangle$ is the state defined at the end of Sect. II.A, and $G_{2 k}(\gamma)$ is the sum of all Feynman graphs of order $2 k$ in $\gamma$, where the interaction is $\sum_{i=2}^{l}(-1)^{i+1} a_{2 i} \beta^{i} \gamma^{2 i}: \phi^{2 i}:_{m}$ as in (19) and the mass is given also by (19). There are $n_{x}$ external legs at each $x \in \mathbb{Z}^{d}$.

Remarks. 1) The Wick-ordering of the interaction is closely related to the " $\alpha$ trick" of $[14,15]$ and other renormalization procedures [11-13].

2) A similar theorem holds if we fix $\beta \geqq 1$.

\section{General Properties of the Models}

1. Infrared bounds. a) $G(p)$ is defined via

$$
\left\langle\phi_{0} \phi_{x}\right\rangle=(2 \pi)^{-3} \int_{-\pi}^{\pi} d^{3} p \exp (i p \cdot x) G(p)
$$


then $G(p)=C \delta(p)+F(p)$ and

$$
F(p) \leqq\left[2 \sum_{\alpha=1}^{3}\left(1-\cos p_{\alpha}\right)\right]^{-1} .
$$

b) As shown in $[27,28]$,

$$
\left\langle\phi_{0} \phi_{x}\right\rangle-C \leqq \text { Const }|x|^{-1} \text {. }
$$

2. Integration by parts (IP). For any $m>0$ and any function such that $\left\langle F^{2}\right\rangle$, $\left.\left\langle d F / d \phi_{x}\right)^{2}\right\rangle<\infty$ for all $x$, we have

$$
\begin{aligned}
\left\langle\phi_{0} F\right\rangle= & \sum_{y \in \mathbb{Z}^{d}} C_{0 y}^{m}\left\langle d / d \phi_{y} F\right\rangle \\
& +\sum_{y \in \mathbb{Z}^{d}} C_{0 y}^{m}\left\langle F\left(d / d \phi_{y}\right) V\left(\phi_{y}\right)\right\rangle+m^{2} \sum_{y \in \mathbb{Z}^{d}} C_{0 y}^{m}\left\langle F \phi_{y}\right\rangle,
\end{aligned}
$$

where

$$
V\left(\phi_{x}\right)=-\frac{\lambda}{4} \phi_{x}^{4}+\frac{\sigma}{2} \phi_{x}^{2}
$$

in the $\phi^{4}$ case and

$$
V\left(\phi_{x}\right)=-\frac{\gamma^{2}}{2} \phi_{x}^{2}+\ln \cosh \sqrt{\beta} \gamma \phi
$$

in the other case.

3. States $\left\langle>\right.$ for the $\phi^{4}$ and $\log \cosh$ theories satisfy the FKG, GHS [29] and Gaussian inequalities [25,26].

Remark. In all the proofs we shall use without comment a number of estimates on sums of Gaussian covariances. For example,

and

$$
\sum_{x \in \mathbb{Z}^{d}} C_{0 x}^{m}=m^{-2}, \quad \sum_{x, y \in \mathbb{Z}^{3}} C_{0 x}^{m} C_{0 y}^{m} C_{x y}=0\left(m^{-3}\right)
$$

$$
\sum_{x \in \mathbb{Z}^{3}} C_{0 x}^{m}(|x|+1)^{-1}=0\left(m^{-1}\right) \quad \text { as } \quad m \rightarrow 0 .
$$

$\sum_{x \in \mathbb{Z}^{3}}\left(C_{0 x}^{m}\right)^{2}$ is also of order $m^{-1}$ as $m \rightarrow 0$ or $C_{00}-C_{00}^{m} \leqq c m$ as $m \rightarrow 0$.

All these estimates can be checked by using Fourier transforms.

\section{Proof of Theorem 1}

1) The upper bound on $T_{c}$ (part $a$ ) is simply Fisher's upper bound [2] $T_{c}(d)$ $\leqq 2 d-1$. For the lower bound (b) the infrared bounds [1] show that if $T<C_{00}^{-1}$ there is long range order. So we have to estimate $2 d C_{00}(d)$. This was done in [32] but one can also compute it as follows:

$$
2 d C_{00}(d)=(2 \pi)^{-d} \int_{-\pi}^{\pi} \frac{d^{d} k}{1-\left(\sum_{\alpha=1}^{d} \frac{\cos k_{\alpha}}{d}\right)} ;
$$


we expand the denominator in the integrand, using

$$
1 / 1-x=1+x+x^{2}+x^{3}+0\left(x^{4}\right)
$$

compute the term $x^{2}$ the $x$ and $x^{3}$ terms vanish) and check that the fourth order term is $0\left(d^{-2}\right)$ as $d \rightarrow \infty$.

2) The upper bound also follows from Fisher's [2] or Griffith's [33] mean field bounds on $T_{c}$. Indeed there is exponential clustering as long as

$$
\beta \sum_{x \neq 0} J_{0 x}<1
$$

but

$$
\begin{aligned}
\sum_{x \neq 0} J_{0 x} & =\sum_{x \in \mathbb{Z}^{d}} J_{0 x}-J_{00} \\
& =1-\gamma^{2} C_{00} \\
& =1-\gamma^{2} C_{00}+o\left(\gamma^{2}\right) .
\end{aligned}
$$

Part b) is an immediate consequence of the infrared bounds. Indeed following the lines of example 5.3 of [34] it is easy to check that $J_{x y}(\gamma)$ is reflection positive. One can then use a generalization of Proposition 3.3 of [35] or [36] and the translation invariance of the state \langle\rangle$(\beta)$ to prove the validity of the infrared bounds for this state: If $G(p)$ is the Fourier transform of $\left\langle\sigma_{0} \sigma_{x}\right\rangle(\beta)$ then the infrared bounds read

$$
G(p)=C \delta(p)+F(p)
$$

and

$$
F(p) \leqq(\beta(J(0)-J(p)))^{-1},
$$

where $J(p)$ is the Fourier transform of $J_{0 x}$. Obviously, $C \neq 0$ if

$$
1>(2 \pi)^{-d} \int_{-\pi}^{\pi} \frac{d^{d} p}{\beta(J(0)-J(p))}
$$

or

$$
\beta>(2 \pi)^{-d} \int_{-\pi}^{\pi} \frac{d^{d} p}{1-\gamma^{2}\left(2 \sum_{e}\left(1-\cos p_{e}\right)+\gamma^{2}\right)^{-1}}=1+\gamma^{2} C_{00}
$$

\section{Proof of Theorem 2}

In Lemma 1 below, we prove estimates on $\left\langle\phi_{0}^{2}\right\rangle$ and $\left\langle\exp \phi_{0}\right\rangle$. Although these quantities are bounded for fixed $\lambda, \sigma$ by superstability (or chessboard) estimates, we shall need shaper bounds, uniform in $\lambda, \sigma$ as long as $\sigma / \lambda$ is bounded.

\section{Lemma 1.}

a) For all $\lambda, \sigma$

$$
\frac{\sigma}{3 \lambda} \leqq\left\langle\phi^{2}\right\rangle \leqq C_{00}+\frac{\sigma}{\lambda}
$$


b) For $\sigma<3 \lambda C_{00}\left\langle: \phi_{0}^{2}:_{m}\right\rangle \geqq 0$, where $m$ is defined by (16).

Proof. The upper and lower bounds in a) are based on one IP :

$$
\begin{aligned}
\left\langle\phi_{0}^{2}\right\rangle= & C_{00}^{m_{0}}+\sum_{x \in \mathbb{Z}^{3}} C_{0 x}^{m_{0}}\left\langle\phi_{0}\left(-\lambda \phi_{x}^{3}+\sigma \phi_{x}\right)\right\rangle \\
& +m_{0}^{2} \sum_{x \in \mathbb{Z}^{3}} C_{0 x}^{m_{0}}\left\langle\phi_{0} \phi_{x}\right\rangle
\end{aligned}
$$

We start with the upper bound in a).

By Griffith's inequalities

$$
\left\langle\phi_{0} \phi_{x}^{3}\right\rangle \geqq\left\langle\phi_{0} \phi_{x}\right\rangle\left\langle\phi_{0}^{2}\right\rangle \text { and }\left\langle\phi_{0} \phi_{x}\right\rangle \geqq 0 .
$$

So,

$$
\begin{aligned}
\left\langle\phi_{0}^{2}\right\rangle \leqq & C_{00}^{m_{0}}+\sum_{x \in \mathbb{Z}^{3}} C_{0 x}^{m_{0}}\left\langle\phi_{0} \phi_{x}\right\rangle\left(-\lambda\left\langle\phi_{0}^{2}\right\rangle+\sigma\right) \\
& +m_{0}^{2} \sum_{x \in \mathbb{Z}^{3}} C_{0 x}^{m_{0}}\left\langle\phi_{0} \phi_{x}\right\rangle .
\end{aligned}
$$

Finally

$$
\begin{aligned}
&\left\langle\phi_{0}^{2}\right\rangle \leqq \frac{C_{00}^{m_{0}}+\left(\sigma+m_{0}^{2}\right) \sum_{x} C_{0 x}^{m_{0}}\left\langle\phi_{0} \phi_{x}\right\rangle}{1+\lambda \sum_{x} C_{0 x}^{m_{0}}\left\langle\phi_{0} \phi_{x}\right\rangle} \\
&\left\langle C_{00}+\sigma / \lambda\left(\text { letting } m_{0} \rightarrow 0\right) .\right.
\end{aligned}
$$

For the lower bound in a) we consider the case $\sigma \geqq 3 \lambda C_{00}$ because the other case follows from b) which is derived below. Using in (23) $\left\langle\phi_{0} \phi_{x}\right\rangle \geqq 0$, and Lebowitz' inequality:

$$
\left\langle\phi_{0} \phi_{x}^{3}\right\rangle \leqq 3\left\langle\phi_{0} \phi_{x}\right\rangle\left\langle\phi_{0}^{2}\right\rangle
$$

we have

$$
\left\langle\phi_{0}^{2}\right\rangle \geqq C_{00}^{m_{0}}+\sum_{x \in \mathbb{Z}^{3}} C_{0 x}^{m_{0}}\left\langle\phi_{0} \phi_{x}\right\rangle\left(-3 \lambda\left\langle\phi_{0}^{2}\right\rangle+\sigma\right) .
$$

Now assume that $-3 \lambda\left\langle\phi_{0}^{2}\right\rangle+\sigma>0$, then, the sum above is strictly positive, for $\left\langle\phi_{0} \phi_{x}\right\rangle>0$, and therefore $\left\langle\phi_{0}^{2}\right\rangle>C_{00}$ (letting $m_{0} \rightarrow 0$ ). But, by the IR bounds, this implies

$$
\lim _{|x| \rightarrow \infty}\left\langle\phi_{0} \phi_{x}\right\rangle \neq 0
$$

and therefore

$$
\lim _{m_{0} \rightarrow 0} \sum_{x \in \mathbb{Z}^{3}} C_{0 x}^{m_{0}}\left\langle\phi_{0} \phi_{x}\right\rangle=+\infty
$$

This contradicts the boundedness of $\left\langle\phi_{0}^{2}\right\rangle$, so $-3 \lambda\left\langle\phi_{0}^{2}\right\rangle+\sigma \leqq 0$.

Part b) is a result of [4]. 
Remarks. 1. By a result due to Newman [30], Proposition 1 implies $\left\langle\exp \phi_{0}\right\rangle$ $\leqq \exp \left(c\left\langle\phi_{0}^{2}\right\rangle\right)$ and $\left\langle\left|\phi_{0}\right|^{n}\right\rangle \leqq n\left(\left\langle\phi_{0}^{2}\right\rangle\right)^{n / 2}$.

2. These bounds on $\langle\exp \phi\rangle$ can be combined with the decay of the truncated two-point function in (21) and FKG inequalities as in [28] to prove the following decay of all truncated correlation functions:

$$
0 \leqq\left\langle\phi_{A} \phi_{B}\right\rangle-\left\langle\phi_{A}\right\rangle\left\langle\phi_{B}\right\rangle \leqq \frac{(\ln \operatorname{dist}(A, B))^{p}}{(\operatorname{dist}(A, B))}
$$

where $\phi_{A}=\prod_{i \in A} \phi_{i}^{n_{i}}$ and $p=\left(\sum_{i \in A} n_{i}+\sum_{i \in B} n_{i}^{\prime}-2\right)$.

Lemma 2. For $\sigma(\lambda)=\left(3 C_{00}-\varepsilon\right) \lambda$, with $\varepsilon>0$,

$$
0 \leqq\left\langle\phi_{0}\right\rangle \leqq 0\left(\lambda^{1 / 2}|\log \lambda|^{3}\right) \quad \text { as } \quad \lambda \rightarrow 0 .
$$

Proof. Let $m$ be defined by (17). Then IP gives

$$
\left\langle\phi_{0}\right\rangle=-\frac{\lambda}{m^{2}}\left\langle: \phi_{0}^{3}:_{m}\right\rangle
$$

since

$$
\sum_{x \in \mathbb{Z}^{3}} C_{0 x}^{m}=m^{-2}
$$

and

$$
\begin{aligned}
\left\langle: \phi_{0}^{3}:_{m}\right\rangle= & -\lambda \sum_{x \in \mathbb{Z}^{3}} C_{0 x}\left\langle: \phi_{0}^{2}:_{m}: \phi_{x}^{3}:_{m}\right\rangle \\
= & -\lambda \sum_{x \in \mathbb{Z}^{3}} C_{0 x}\left(\left\langle: \phi_{0}^{2}:_{m}: \phi_{x}^{3}:_{m}\right\rangle-\left\langle: \phi_{0}^{2}:_{m}\right\rangle\left\langle: \phi_{x}^{3}:_{m}\right\rangle\right) \\
& +\left\langle: \phi_{0}^{2}:_{m}\right\rangle\left\langle\phi_{0}\right\rangle
\end{aligned}
$$

using (26).

Inserting (27) in (26) one finds

$$
\left\langle\phi_{0}\right\rangle\left(\frac{m^{2}}{\lambda}+\left\langle: \phi_{0}^{2}:_{m}\right\rangle\right)=\lambda \sum_{x \in \mathbb{Z}^{3}} C_{0 x}\left(\left\langle: \phi_{0}^{2}:_{m}: \phi_{x}^{3}:_{m}\right\rangle-\left\langle: \phi_{0}^{2}:_{m}\right\rangle\left\langle: \phi_{x}^{3}:_{m}\right\rangle\right) .
$$

By Lemma 1.b, $\left\langle: \phi_{0}^{2}:_{m}\right\rangle \geqq 0$ and by hypothesis $m^{2} / \lambda$ is bounded from below. By the bound (25), we know that

$$
\left\langle: \phi_{0}^{2}:_{m}: \phi_{x}^{3}:_{m}\right\rangle-\left\langle: \phi_{0}^{2}:_{m}\right\rangle\left\langle: \phi_{x}^{3}:_{m}\right\rangle \mid
$$

is of order $(\log |x|)^{3} /|x|$, which implies that the right hand side of $(28)$ is smaller than $\lambda / m|\log m|^{3} \simeq \sqrt{\lambda}|\log \lambda|^{3}(m$ is of order $\sqrt{\lambda}$ ).

This lemma is only used for the proof of the stronger

Lemma 3. For $\sigma(\lambda)=\left(3 C_{00}-\varepsilon\right) \lambda$ with $\varepsilon>0$ and $\lambda$ small enough $\left\langle\phi_{0}\right\rangle=0$.

Proof. By IP with respect to $m(\lambda)$ :

$$
\left\langle\phi_{0}\right\rangle=-\lambda / m^{2}\left\langle: \phi_{0}^{3}:\right\rangle
$$


and

$$
\begin{aligned}
\left\langle: \phi_{0}^{3}:\right\rangle & =-\lambda \sum_{x} C_{0 x}^{m}\left\langle: \phi_{0}^{2}:: \phi_{x}^{3}:\right\rangle \\
& =-3 \lambda \sum_{x}\left(C_{0 x}^{m}\right)^{2}\left\langle\phi_{0}: \phi_{x}^{2}:\right\rangle+\lambda^{2} \sum_{x, y} C_{0 x}^{m} C_{0 y}^{m}\left\langle\phi_{0}: \phi_{x}^{3}:: \phi_{y}^{3}:\right\rangle, \\
\left\langle\phi_{0}: \phi_{x}^{2}:\right\rangle & =\left\langle\phi_{0} \phi_{x}^{2}\right\rangle-C_{00}^{m}\left\langle\phi_{0}\right\rangle \\
& \leqq 2\left\langle\phi_{0} \phi_{x}\right\rangle\left\langle\phi_{0}\right\rangle+\left\langle\phi_{0}\right\rangle\left\langle\phi_{0}^{2}\right\rangle-C_{00}^{m}\left\langle\phi_{0}\right\rangle
\end{aligned}
$$

by the GHS inequality [29]. So the first term is bounded by

$$
0\left(\frac{\lambda}{m}\right)\left\langle\phi_{0}\right\rangle \leqq 0(\sqrt{\lambda})\left\langle\phi_{0}\right\rangle .
$$

Moreover,

$$
\left\langle\phi_{0}: \phi_{x}^{3}:: \phi_{y}^{3}:\right\rangle
$$

is bounded, by the Gaussian inequality (28), by a sum of terms, each of which is a product containing at least $\left\langle\phi_{0}\right\rangle$ and one $\left\langle\phi_{0} \phi_{x}\right\rangle$ or $\left\langle\phi_{0} \phi_{y}\right\rangle$ or $\left\langle\phi_{x} \phi_{y}\right\rangle$. Writing

$$
\left\langle\phi_{0} \phi_{x}\right\rangle=\left(\left\langle\phi_{0} \phi_{x}\right\rangle-\left\langle\phi_{0}\right\rangle^{2}\right)+\left\langle\phi_{0}\right\rangle^{2} \leqq c /|x|+\left\langle\phi_{0}\right\rangle^{2}
$$

we get a sum of terms which are either of order $\left\langle\phi_{0}\right\rangle \lambda^{2} / \mathrm{m}^{3}$ or $\lambda^{2} / \mathrm{m}^{4}\left\langle\phi_{0}\right\rangle^{3}$; so for $m^{2}$ of order $\lambda$

$$
\left(\phi_{0}\right\rangle \leqq\left(c_{1}(\sqrt{\lambda})\left\langle\phi_{0}\right\rangle+c_{2}\left\langle\phi_{0}\right\rangle^{3}\right)
$$

or

$$
\left(1-c_{1} \sqrt{\lambda}\right)\left\langle\phi_{0}\right\rangle \leqq c_{2}\left\langle\phi_{0}\right\rangle^{3} .
$$

Now, if $\left\langle\phi_{0}\right\rangle$ were nonzero then, $\left(1-c_{1} \sqrt{\lambda}\right) \leqq c_{2}\left\langle\phi_{0}\right\rangle^{2}$. But since $\left\langle\phi_{0}\right\rangle \rightarrow 0$ as $\lambda \rightarrow 0$ (Lemma 2), this is impossible.

Now we prove Theorem 2. Part a) is based on Theorem 3 (see Sect. V). However, in the proof of Theorem 3, we use the infrared bounds with $C=0$ in (21). This is precisely Lemma 3 since the state $\left\langle>\right.$ of the $\phi^{4}$ theory is clustering [21]. Proof of Theorem 2. By Simon's inequalities [6] we know that there is exponential clustering of $\left\langle\phi_{0} \phi_{x}\right\rangle$ as soon as

$$
\sum_{x \in \partial A}\left\langle\phi_{0} \phi_{x}\right\rangle<1 / 2
$$

for some box $\Lambda$ with the sum running over the boundary of $\Lambda$. We shall choose the radius of $\Lambda$ to depend on $\lambda$ in such a way that the sum (29) goes to zero as $\lambda$ goes to zero. Specifically, we shall show that

$$
\lim _{\lambda \rightarrow 0} \lambda^{-(1+\varepsilon)}\left\langle\phi_{0} \phi_{x(\lambda)}\right\rangle=0,
$$

where $|x(\lambda)| \simeq 0\left(\lambda^{-1 / 2-\varepsilon / 2}\right)$ for some $\varepsilon>0(|\partial \Lambda|$ is of the order of the square of the radius of 1 ), and this clearly implies the result. Now (30) follows from the expansion of Theorem 3

$$
\left\langle\phi_{0} \phi_{x(\lambda)}\right\rangle=\sum_{k=0}^{2} G_{k}(\lambda)+R_{2}(\lambda) \lim _{\lambda \rightarrow 0} \lambda^{-(1+\varepsilon)} \sum_{k=0}^{2} G_{k}(\lambda)=0,
$$


because in each $G_{k}(\lambda)$ there is a factor $\exp (-m|x|) \sim \exp \left(-\lambda^{-\varepsilon}\right)$. Moreover the proof of Theorem 3 implies that the bound on $R_{2}(\lambda)$ is independent of $x(\lambda)$, i.e. $\lim _{\lambda \rightarrow 0} \lambda^{-2} R_{2}(\lambda)=0$.

Theorem $2 b$ is an immediate consequence of the infrared bounds and of Lemma 1a.

Remark. For polynomials of higher degree (with positive coefficients) it is easy to show

$$
\sigma_{c}(\lambda)=2 \lambda \sum_{i=1}^{n}\left((2 i) ! a_{i} /(i-1) ! 2^{i}\right) C_{00}^{i-1}
$$

to first order in $\lambda$, at least if $\sigma_{c}(\lambda)$ means the point where $\langle\phi\rangle$ vanishes. We proceed as follows: the proof of Lemma 1a extends to show that $\left\langle\phi_{0}^{2}\right\rangle\langle\infty$ as long as $\sigma / \lambda$ is bounded. On the one hand, if

$$
\sigma>2 \lambda \sum_{i=2}^{n} \frac{(2 i) ! a_{i}}{(i-1) ! 2^{i}}\left(C_{00}\right)^{i-1}
$$

then, we use the Gaussian inequality

$$
\left\langle\phi_{0} \phi_{x}^{2 i-1}\right\rangle \leqq \frac{(2 i) !}{(i-1) ! 2^{i-1}}\left\langle\phi_{0} \phi_{x}\right\rangle\left\langle\phi_{0}^{2}\right\rangle^{i-1},
$$

which in turn implies $\left\langle\phi_{0}^{2}\right\rangle>C_{00}$ as in the proof of Lemma 1, and this implies long range order. On the other hand, in the proof of Lemma 2, we get instead of (28)

$$
\left.\mid\left\langle\phi_{0}\right\rangle \frac{m^{2}}{\lambda}+\left\langle: \bar{V}^{\prime}(\phi):_{m}\right\rangle\right) \mid \leqq 0\left(\lambda^{1 / 2}|\ln \lambda|^{p}\right),
$$

where

$$
: V(\phi):=\sum_{i=2}^{n} b_{i}: \phi^{2 i}:
$$

is the Wick-ordering of $\sum_{i=2}^{n} a_{i} \phi^{2 i}$ (up to a constant), and

$$
: \bar{V}^{\prime}(\phi):=\sum_{i=2}^{n} b_{i} 2 i: \phi_{i}^{2 i-2}: .
$$

By integrating by parts all the powers of $\phi$, we show that

$$
\left\langle: \phi_{0}^{2 h}:\right\rangle \geqq-0(\sqrt{\lambda}) \quad(h \in N)
$$

and this is sufficient to conclude Lemma 2 . If we integrate by parts $\left\langle: \phi_{0}^{2 h}:\right\rangle$, all the Gaussian terms are small $0(\lambda)$. The worst term in the remainder is

$$
\lambda^{2 h} \sum_{x_{1} \ldots x_{2 h}} C_{0 x_{1}} \ldots C_{0 x_{2 h}}\left\langle: V^{\prime}\left(\phi_{x_{1}}\right):_{m} \ldots: V^{\prime}\left(\phi_{x_{2 h}}\right):_{m}\right\rangle,
$$

but this is positive. All the other terms contain some contraction, i.e. some additional line $C_{x_{i} x_{j}}$, and are therefore at worst of order $m \sim \sqrt{\lambda}$. The proof of Lemma 3 then follows as in the $\lambda \phi^{4}$ case. 


\section{Perturbation Theory}

We only give the proof of Theorem 4; the proof of Theorem 3 is included in it, using the truncation (13). We first need a bound on $\left\langle\phi_{0}^{2}\right\rangle$ for the $\log \cosh \phi$ theory.

A) Bounds on $\left\langle\phi_{0}^{2}\right\rangle$

Proposition. Let $\beta-1=b \gamma^{2}$, then $\forall \alpha>0, \sup _{\gamma \in[0, \alpha]}\left\langle\phi_{0}^{2}\right\rangle<\infty$.

The proof of this proposition will use the FKG inequalities and the following:

Lemma 4. Let $g(x)$ be defined by $g(x)=x^{3} / 6|x| \leqq 1, g(x)=x / 6|x|>1$, then

$$
h(x)=\tanh x-x+g(x)
$$

is a decreasing function of $x$.

The proof is straightforward: we just have to show $h^{\prime}(x) \leqq 0$. We use the fact that $1 / 6<1-\cosh ^{-2} 1$ and that $\cosh ^{-2} x \leqq 1-x^{2} / 2$ for $|x| \leqq 1$. Let us introduce $\chi_{>}(\phi)$, the characteristic function of the set $|\sqrt{\beta} \gamma \phi|>1, \chi_{<}=1-\chi_{>}$.

Proof of Proposition. The proof is divided in two steps. First we prove that $\left|\phi_{0}\right|$ has a very small probability of being large (of order $\gamma^{-1}$ ): we show that $\left.\left\langle\chi_{>}(\phi)_{0}\right)\right\rangle$ is of order $\gamma^{2}$ as $\gamma \rightarrow 0$ (in a)). But then, because of Lemma 4, we know that the quartic approximation to $\log \cosh x$ is good when $|\phi| \leqq 1 / \sqrt{\beta} \gamma$ and we can (almost) use the proof done for $\phi^{4}$ (Lemma 1): see (b) below.

a) The IP formula with respect to a covariance of mass $m^{2}=a \gamma^{4}$ gives :

$$
\begin{aligned}
\left\langle\phi_{0}^{2}\right\rangle= & C_{00}^{m}-\gamma^{2} \sum_{x} C_{0 x}^{m}\left\langle\phi_{0} \phi_{x}\right\rangle+\sqrt{\beta} \gamma \sum_{x} C_{0 x}^{m}\left\langle\phi_{0} \tanh \sqrt{\beta} \gamma \phi_{x}\right\rangle \\
& +m^{2} \sum_{x} C_{0 x}^{m}\left\langle\phi_{0} \phi_{x}\right\rangle .
\end{aligned}
$$

The FKG inequalities together with the Lemma give $(x=\sqrt{\beta} \gamma \phi)$

$$
\begin{aligned}
& \left\langle\phi_{0}\left(\tanh \sqrt{\beta} \gamma \phi_{x}-\sqrt{\beta} \gamma \phi_{x}+g\left(\sqrt{\beta} \gamma \phi_{x}\right)\right)\right\rangle \\
& \quad \leqq\left\langle\phi_{0}\right\rangle\left\langle\tanh \sqrt{\beta} \gamma \phi_{x}-\sqrt{\beta} \gamma \phi_{x}+g\left(\sqrt{\beta} \gamma \phi_{x}\right)\right\rangle=0 .
\end{aligned}
$$

This and (31) give:

$$
\begin{aligned}
\left\langle\phi_{0}^{2}\right\rangle \leqq & C_{00}^{m}-\sum_{x} C_{0 x}^{m} \frac{\beta^{2} \gamma^{4}}{6}\left\langle\phi_{0} \phi_{x}^{3} \chi_{<}\right\rangle \\
& -\sum_{x} C_{0 x}^{m} \frac{\beta \gamma^{2}}{6}\left\langle\phi_{0} \phi_{x} \chi_{>}\right\rangle \\
& +d \gamma^{4} \sum_{x} C_{0 x}^{m}\left\langle\phi_{0} \phi_{x}\right\rangle,
\end{aligned}
$$

where $d=b+a, b=\beta-1 / \gamma^{2}$. A generalized form of Griffith's inequality (Nelson's inequalities [22]) imply:

$$
\left\langle\phi_{0} \phi_{x} \chi_{>}\right\rangle \geqq\left\langle\phi_{0} \phi_{x}\right\rangle\left\langle\chi_{>}\right\rangle \text {. }
$$


This combined with (32) yields

$$
\left\langle\phi_{0}^{2}\right\rangle \leqq C_{00}^{m}-\sum_{x} C_{0 x}^{m} \frac{\beta \gamma^{2}}{6}\left\langle\phi_{0} \phi_{x}\right\rangle\left\langle\chi_{>}\right\rangle+d \gamma^{4} \sum_{x} C_{0 x}^{m}\left\langle\phi_{0} \phi_{x}\right\rangle .
$$

Now assume that $\sup _{\gamma \in[0, \alpha]}\left\langle\phi_{0}^{2}\right\rangle=\infty$. Since $\left\langle\phi_{0}^{2}\right\rangle$ is bounded for any $\gamma \neq 0$, it means that there exists a sequence $\gamma_{n} \rightarrow 0$ for which $\left\langle\phi_{0}^{2}\right\rangle\left(\gamma_{n}\right)$ diverges to $+\infty$. But, by (33), as soon as $\left\langle\phi_{0}^{2}\right\rangle>C_{00}^{m}$ we must have

$$
\frac{\beta \gamma^{2}}{6}\left\langle\chi_{>}\right\rangle \leqq d \gamma^{4},
$$

or

$$
\left\langle\chi>\left(\phi_{0}\right)\right\rangle \leqq \text { const } \gamma^{2} .
$$

If $\left\langle\phi_{0}^{2}\right\rangle(\gamma)$ was bounded uniformly in $\gamma$, (34) would follow from Chebyshev's inequality. So (34) holds in either case.

b) We now rewrite (32) using $\chi_{<}=1-\chi_{>}$and $\left\langle\phi_{0} \phi_{x}^{3}\right\rangle \geqq\left\langle\phi_{0} \phi_{x}\right\rangle\left\langle\phi_{0}^{2}\right\rangle$ :

$$
\begin{aligned}
\left\langle\phi_{0}^{2}\right\rangle \leqq & {\left[C_{00}^{m}+\sum_{x} C_{0 x}^{m} \frac{\beta^{2} \gamma^{4}}{6}\left\langle\phi_{0} \phi_{x}^{3} \chi_{>}\right\rangle+d \gamma^{4} \sum_{x} C_{0 x}^{m}\left\langle\phi_{0} \phi_{x}\right\rangle\right] } \\
& \cdot\left[1+\frac{\beta^{2} \gamma^{4}}{6} \sum_{x} C_{0 x}^{m}\left\langle\phi_{0} \phi_{x}\right\rangle\right]^{-1} \\
\left\langle\phi_{0}^{2}\right\rangle \leqq & C_{00}^{m}+\frac{6 d}{\beta^{2}}+\left[\sum_{x} C_{0 x}^{m} \frac{\beta^{2} \gamma^{4}}{6}\left\langle\phi_{0} \phi_{x}^{3} \chi_{>}\right\rangle\right] \\
& \cdot\left[1+\sum_{x} C_{0 x}^{m} \frac{\beta^{2} \gamma^{4}}{6}\left\langle\phi_{0} \phi_{x}\right\rangle\right]^{-1} .
\end{aligned}
$$

Using Schwartz's inequality together with the Gaussian inequality,

$$
\left\langle\phi_{0} \phi_{x}^{3} \chi_{>}\right\rangle \leqq\left\langle\chi_{>}\right\rangle^{1 / 2} \text { Const }\left\langle\phi_{0}^{2}\right\rangle^{2}
$$

(35) gives:

$$
\left\langle\phi_{0}^{2}\right\rangle \leqq C_{00}^{m}+\frac{6 d}{\beta^{2}}+\frac{\operatorname{const}\left\langle\phi_{0}^{2}\right\rangle^{2}\left\langle\chi_{>}\right\rangle^{1 / 2}}{1+\sum_{x} C_{0 x}^{m} \frac{\beta^{2} \gamma^{4}}{6}\left\langle\phi_{0} \phi_{x}\right\rangle}
$$

because $\sum_{x} C_{0 x}=\left(a \gamma^{4}\right)^{-1}$. From this it follows that if $\left\langle\phi_{0}^{2}\right\rangle$ is larger than $C_{00}^{m}+\frac{6 d}{\beta^{2}}$ (in particular, if it diverges as $\gamma \rightarrow 0$ ), then

$$
\gamma^{4} \sum_{x} C_{0 x}^{m}\left\langle\phi_{0} \phi_{x}\right\rangle \leqq \text { const }\left\langle\phi^{2}\right\rangle\left\langle\chi_{>}\right\rangle^{1 / 2}
$$

(multiply both sides of (36) by $\left.1+\sum_{x} C_{0 x}^{m} \frac{\beta^{2} \gamma^{2}}{6}\left\langle\phi_{0} \phi_{x}\right\rangle\right)$. By (33)

$$
\left\langle\phi_{0}^{2}\right\rangle \leqq C_{00}^{m}+d \gamma^{4} \sum_{x} C_{0 x}^{m}\left\langle\phi_{0} \phi_{x}\right\rangle .
$$


Using (37) we have for any sequence $\left(\gamma_{n}\right)$ such that $\left\langle\phi_{0}^{2}\right\rangle\left(\gamma_{n}\right)$ diverges :

For $\gamma$ small

$$
\begin{aligned}
\left\langle\phi_{0}^{2}\right\rangle & \leqq C_{00}^{m}+\text { Const }\left\langle\phi_{0}^{2}\right\rangle\left\langle\chi_{>}\right\rangle^{1 / 2} \\
& \leqq C_{00}+\text { Const }\left\langle\phi_{0}^{2}\right\rangle \gamma \quad[b y(34)] .
\end{aligned}
$$

$$
\left\langle\phi_{0}^{2}\right\rangle \leqq C_{00}[1 \text {-const } \gamma]^{-1},
$$

but this is bounded as $\gamma \rightarrow 0$ so $\left\langle\phi_{0}^{2}\right\rangle$ cannot diverge.

Remark. By [30] $\left\langle\left|\phi_{0}\right|^{n}\right\rangle\left\langle n !\left(\left\langle\phi_{0}^{2}\right\rangle^{1 / 2}\right)^{n}\right.$.

B. Proof of Theorem 4. To be specific, let us choose

$$
\left\langle\prod_{x} \phi_{x}^{n_{x}}\right\rangle=\left\langle\phi\left(x_{1}\right) \ldots \phi\left(x_{k}\right)\right\rangle \text {. }
$$

Truncation of the interaction. We start by applying Formula (19) with $n=l+2$. The perturbation theory will be generated by the IP formula with respect to the covariance $C_{0 x}^{m}$. Obviously, the contribution of the "remainder" in (19) will always be negligible compared to $\gamma^{2 l}$ because

$$
\begin{aligned}
& \sum_{x} C_{y, x}\left\langle\phi\left(x_{2}\right) \ldots \phi\left(x_{k}\right) \frac{d}{d \phi_{x}} \text { "Remainder" }\right\rangle \\
& \leqq c \gamma^{2 l+6} \gamma^{-4}=0\left(\gamma^{2 l+2}\right) .
\end{aligned}
$$

So it is the equivalent to prove the theorem for the case of a polynomial interaction given by

$$
\sum_{i=2}^{l+2}(-1)^{i+1} a_{2 i} \beta^{i} \gamma^{2 i}: \phi^{2 i}:_{m},
$$

as perturbation of our massive Gaussian measure with $m^{2} \sim \varepsilon \gamma^{4}$.

For this case we apply successively the IP formula until all the terms produced either:

a) are fully contracted, i.e., are purely Gaussian terms,

b) contain $l+1: \phi^{2 i}$ : vertices for $i \in\{3,4,5, \ldots, l+2\}$. Each of these vertices may still have contracted legs in the non-Gaussian expectation values. That is, these terms result from at least $l+1$ derivation of $\exp \gamma^{2 i}: \phi^{2 i}:(2 i \geqq 6)$. Each derivation produces a divergent factor $\sum_{x} C_{x y}=m^{-2}$, and a convergent factor $\gamma^{2 i}$. Therefore these terms are at most of order $\gamma^{2 l+2}$; each additional line which may appear in the diagram due to Gaussian contractions improves the convergence.

c) results from at least $2 l+2 k$ derivatives of $\exp \gamma^{4}: \phi^{4}$ :, where $k$ is the number of external vertices. The proof that these terms are small compared to $\gamma^{2 l}$ contains several steps:

Step 1) We estimate these terms by undoing the Wick-ordering in the nonGaussian expectation values, taking absolute values and applying Gaussian inequalities to produce extra $\langle\phi(x) \phi(y)\rangle$-lines in the diagram. Since we are in the one phase region, (here we use Theorem 1, 2a, and Lemma 3) the infrared bounds (20) imply that each of these new lines corresponds in momentum space to a propagator bounded by $c / p^{2}$. 
More precisely, let us consider one particular term resulting from the IP procedure. It will be of the form (see Fig. 1):

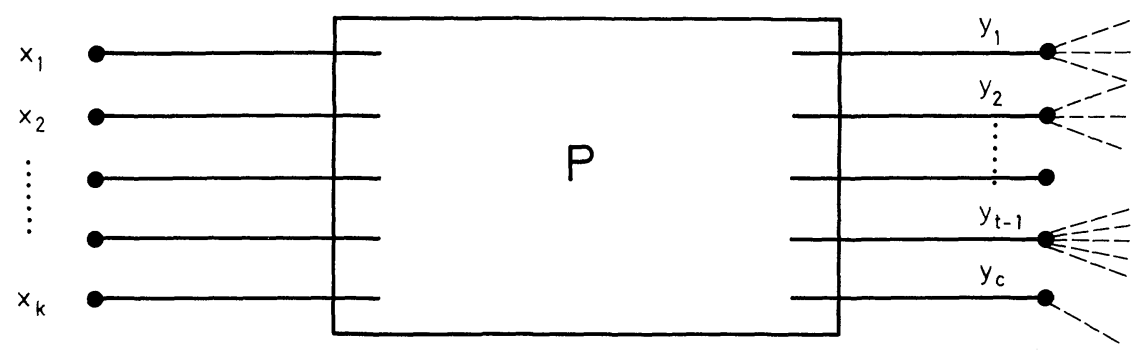

Fig. 1

$$
\sum_{y_{1} \ldots y_{t}} P\left(x_{1}, \ldots, x_{k}, y_{1}, \ldots, y_{t}\right)\left\langle: \phi^{3}:\left(y_{1}\right): \phi^{3}:\left(y_{2}\right) \ldots: \phi^{5}:\left(y_{t-1}\right) \phi\left(y_{t}\right)\right\rangle,
$$

where $P$ is an ordinary Feynman graph except that the legs represented by a dashed line are not yet contracted; they still are part of the non-Gaussian expectation value.

By application of the Gaussian inequalities, this graph is replaced by a sum of other graphs (see Fig. 2):

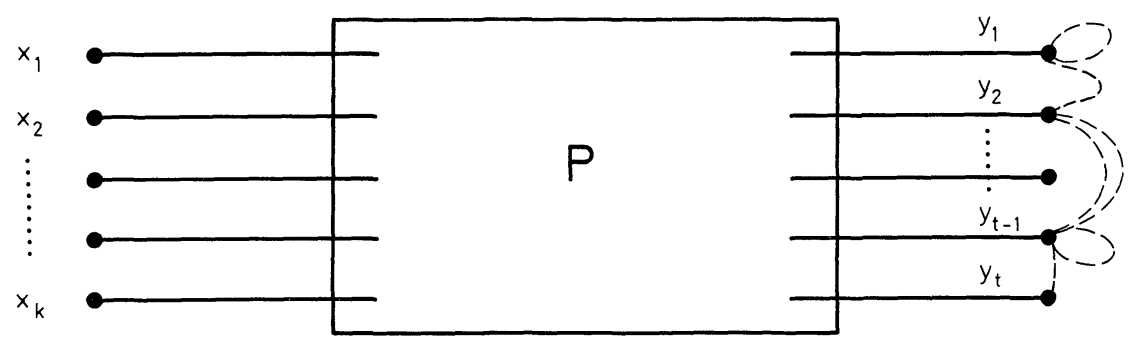

Fig. 2

(after application of 1).

Here --- is a line with propagator $1 / p^{2}$.

Step 2) We now want to estimate all the diagrams produced after application of 1).

Let us call $F\left(x_{1}, \ldots, x_{k}\right)$ the contribution of one such diagram $G$. We denote its $: \phi^{4}:$ vertices by $w_{1}, \ldots, w_{2 l+2 k}$. Here $L\left(w_{i}\right)$ is the number of internal lines which end at $w_{i}$ and $L_{E}\left(w_{i}\right)$ is the number of external lines which end at $w_{i}$. The important consequence of 1) is the following inequality:

$$
\sum_{i}\left[L_{E}\left(w_{i}\right)+L\left(w_{i}\right)\right]-2 l+2 k \geqq l+k .
$$

i) In order to prove (38) consider first the case where all $: \phi^{4}:$ vertices have three legs in the non-Gaussian expectation value: for instance take

$$
\begin{aligned}
F\left(x_{1} \ldots x_{k}\right)= & \sum P\left(x_{1}, \ldots, x_{k}, y_{1}, \ldots, y_{2 l+2 k}, z_{1}, \ldots, z_{t}\right) \\
& \cdot\left\langle: \phi^{3}\left(y_{1}\right):, \ldots, \phi^{3}\left(y_{2 l+2 k}\right): \phi^{5}\left(z_{1}\right): \ldots: \phi^{9}:\left(z_{t}\right)\right\rangle .
\end{aligned}
$$


By application of 1) we bound the non-Gaussian expectation value by a finite sum of products of two point functions. These give rise to lines. There is at least one line out of each : $\phi^{3}$ : vertex, i.e. at least one line is added for every pair of $: \phi^{3}$ : vertices.

Therefore in this case we see that the application of the Gaussian inequalities has generated $l+k$ new massless lines.

ii) If some $: \phi^{4}$ : vertices have less than three legs in the non-Gaussian expectation value, that means that at least two of their legs have been contracted with two other legs. That is at least two massive lines end at these vertices.

Combining i) and ii) we get (38) because the left hand side of (38) would be zero in a tree graph and we have added at least $l+k$ lines.

Now

$$
|F| \leqq \int_{\pi}^{\pi} \frac{d^{3} p_{1}}{(2 \pi)^{3}}, \ldots, \frac{d^{3} p_{k-1}}{(2 \pi)^{3}}\left|\tilde{F}\left(p_{1}, \ldots, p_{k-1}\right)\right|,
$$

where $\tilde{F}$ is the Fourier transform of $F$. We have to show that $|F|$ is small compared to $\gamma^{2 l}$. We first replace in each propagator

$$
\sum_{\alpha=1}^{3}\left(1-\cos p_{\alpha}\right) \text { by } \sum_{\alpha=1}^{3} p_{\alpha}^{2},
$$

because $\frac{2}{\pi^{2}} p^{2}<1-\cos p<\frac{1}{2} p^{2},|p|<\pi$. To compute the infrared behavior of the graph, we scale all momenta : $p_{\alpha}=m p_{\alpha}^{\prime}$. The idea is to do a power counting in $m$ and then control the possible ultraviolet divergence of the scaled graph. To each vertex $v$ of the graph is associated a power of $\gamma, \gamma^{d(v)}$, when $d(v)$ stands for the degree of the vertex (for instance $d=6$ for a $: \phi^{6}$ : vertex).

We start by doing a power counting in $m=\sqrt{\varepsilon} \gamma^{2}$, using a $\gamma^{4}$ convergence factor at each vertex and disregarding the ultraviolet divergences. We shall show using the theorem of the Appendix, that the remaining powers of $\gamma$ of each vertex $v$, $d(v)-4$, enable us to control ultraviolet divergences.

Step 3) The infrared power counting.

i) For each vertex, there is a divergence factor $m^{-3}(\gamma)$ coming from the $\delta$-function and a $\gamma^{(4)}$ convergence factor which results from the splitting of $d(v)$ (see Step 2)). So, there is a $m^{-1}$ divergence.

ii) Each line brings a factor of convergence $m$ coming from the scaling of $\left(d^{3} k\right) /\left(k^{2}+m^{2}\right)$ (see 2$\left.)\right)$. Let $V$ be the number of internal vertices of the graph, $L$ be the number of internal lines, $L_{E}$ be the number of external lines; if the graph $G$ was a tree graph (after identifying all external vertices), $V=L_{E}+L$, and the resulting power of $m$ after scaling would be zero. However, by (38), $L_{E}+L-V \geqq l+k$. Therefore

$$
\begin{aligned}
|F| \leqq & m^{l+k} \int_{-\pi / m}^{\pi / m} \int_{-\pi / m}^{\pi / m} \frac{d^{3} p_{1}}{(2 \pi)^{3}}, \ldots, \frac{d^{3} p_{k-1}}{(2 \pi)^{3}} H\left(p_{1}, \ldots, p_{k-1}\right) \\
& \cdot\left(p_{1}^{2}+1\right)^{-1}, \ldots,\left(p_{k-1}^{2}+1\right)^{-1}
\end{aligned}
$$

where $H$ is the function obtained after the scaling has been performed on $\tilde{F}$ and after the external legs have been amputated. All propagators in $H$ are of mass 1 with the exception of those obtained by application of 1) which are massless. 
Step 4) The ultraviolet power counting.

Let $N$ be the number of loops and $d(v)$ the degree of the vertex $v$. The following relations are well known [43]

$$
\begin{aligned}
L-N & =V-1, \\
2 L+L_{E} & =\sum_{v} d(v) .
\end{aligned}
$$

The superficial divergence of a graph $G$ in powers of the cutoff $m^{-1}, \omega(G)$ $=3 N-2 L$. Using (40),

$$
\omega(G)=\sum_{v} \frac{(d(v)-3)}{2}+3-L_{E} / 2 .
$$

In our case at each vertex $v$, there is an additional factor of convergence $\gamma^{d(v)-4} \simeq m^{d(v) / 2-2}$ (see Step 2).). Let $\Omega(G)$ be the superficial divergence of $G$, after the factor $m^{d(v) / 2-2}$ is added at each vertex.

$$
\begin{aligned}
\Omega(G) & =\sum_{v} \frac{(d(v)-3)}{2}-\sum_{v} \frac{(d(v)-2)}{2}+3-L_{E} / 2 \\
& =-V+3-L_{E} / 2 .
\end{aligned}
$$

Since our interaction is Wick-ordered, there are no graphs with $V=1$. The only divergent graphs have $V=2, L_{E}=2$ for instance (see Fig. 3):
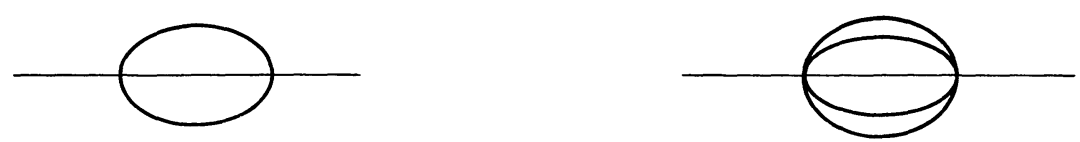

Fig. 3

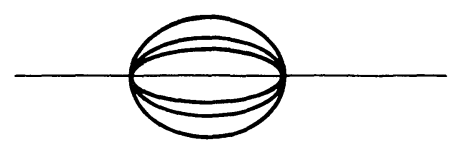

which are only logarithmically divergent $(\Omega(G)=0)$. Therefore by the theorem of the Appendix, all the scaled graphs are at most logarithmically divergent since for all possible subgraphs $H$ of $G \Omega(H)=0$. That is :

$$
\left|H\left(p_{1}, \ldots, p_{k-1}\right)\right| \leqq c(\ln m)^{v} .
$$

This finishes the proof because

$$
\begin{aligned}
& \int_{-\pi / m}^{+\pi / m} \ldots \int_{-\pi / m}^{\pi / m} d^{3} p_{1}, \ldots, d^{3} p_{k-1}\left(p_{1}^{2}+1\right)^{-1}, \ldots,\left(p_{k-1}^{2}+1\right)^{-1}(2 \pi)^{-3 k+3} \\
& \quad \cdot\left|H\left(p_{1}, \ldots, p_{k-1}\right)\right| \leqq C\left(m^{-1}\right)^{(k-1)}(\ln m)^{v} .
\end{aligned}
$$

This combined with (39) implies $|F| \leqq m^{l+1-\varepsilon}, 0<\varepsilon<1$ which is indeed small compared to $\gamma^{2 l}$. 


\section{Remarks and Extensions}

1. Another way to understand our result on $\lambda \phi^{4}$ is to relate it to the continuous $\left(\phi^{4}\right)_{3}$ field theory. Let us write the Hamiltonian of a $\left(\phi^{4}\right)_{3}$ model with all the counterterms except for those which are constant in $\phi$. The ultraviolet cutoff we choose is a lattice spacing cutoff $\delta$.

$$
\begin{aligned}
H= & (1 / 2) \delta \sum_{\langle x, y\rangle}\left(\phi_{x}-\phi_{y}\right)^{2}+\left(\lambda_{0} / 4\right) \delta^{3} \sum_{x} \phi_{x}^{4}-(3 / 2) C_{00}^{m, \delta} \lambda_{0} \delta^{3} \sum_{x} \phi_{x}^{3} \\
& +3\left(\lambda_{0}^{2} / 2\right) \delta^{3} \sum_{y}\left(C_{0 x}^{m, \delta}\right)^{3} \sum_{x} \phi_{x}^{2}+\frac{m^{2}}{2} \delta^{3} \sum_{x} \phi_{x}^{2}-\frac{\alpha^{2}}{2} \delta^{3} \sum_{x} \phi_{x}^{2} .
\end{aligned}
$$

Here $\lambda_{0}$ is the (bare) field theory coupling constant,

$$
C_{00}^{m, \delta}=\delta^{3} /(2 \pi)^{3} \int_{-\pi / \delta}^{\pi / \delta} d^{3} k\left[\delta \sum_{\alpha=1}^{3}\left(1-\cos k_{\alpha} \delta\right)+m^{2} \delta^{3}\right]^{-1},
$$

the $3 \mathrm{rd}$ and 4 th term in the Hamiltonian correspond respectively to the Wick ordering and mass counterterms.

From field theory method we know that

1) if $\alpha=0$ and $\frac{\lambda_{0}}{m}$ small the theory has a mass gap $[38,39]$,

2) if $\frac{\lambda_{0}}{\alpha}$ is small there is long range order [1]. We now want to translate this result into statistical mechanics language. We first rescale $\phi: \sqrt{\delta} \phi \rightarrow \phi^{\prime}$ so that we eliminate the factor $\delta$ in front of the Laplacian:

$$
\begin{aligned}
H^{\prime}= & 1 / 2 \sum_{\langle x, y\rangle}\left(\phi_{x}-\phi_{y}\right)^{2}+\frac{\lambda_{0}}{4} \delta \sum_{x} \phi_{x}^{4}-3 / 2 C_{00}^{m, \delta} \lambda_{0} \delta^{2} \sum_{x} \phi_{x}^{2} \\
& +\frac{3}{2} \lambda_{0}^{2} \delta^{2} \sum_{x}\left(C_{0 y}^{m, \delta}\right)^{3} \sum_{x} \phi_{x}^{2}+\frac{m^{2}}{2} \delta^{2} \sum_{x} \phi_{x}^{2}-\frac{\alpha^{2}}{2} \delta^{2} \sum_{x} \phi_{x}^{2} .
\end{aligned}
$$

Now again by scaling

$$
\begin{aligned}
C_{00}^{m, \delta} & =(2 \pi)^{-3} \int_{-\pi}^{\pi} d^{3} k\left[\delta \sum_{\alpha=1}^{3}\left(1-\cos k_{\alpha}\right)+m^{2} \delta^{3}\right]^{-1} \\
& =\delta^{-1} C_{00}-0(m)
\end{aligned}
$$

and $\sum_{y}\left(C_{0 y}^{m, \delta}\right)^{3}=C \ln (m \delta)+0(1)$, where $C$ is a computable constant.

If we identify

$$
\begin{aligned}
& \lambda=\lambda_{0} \delta, \\
& \sigma=3 \lambda C_{00}-3 C \lambda^{2} \ln \lambda+\lambda^{2}\left[m^{2} / \lambda_{0}^{2}-\alpha^{2} / \lambda_{0}-0\left(m / \lambda_{0}\right)+3 C \ln \frac{m}{\lambda_{0}}+0(1)\right],
\end{aligned}
$$

we recover our lattice model.

1) and 2) mean that

$$
\sigma_{c}(\lambda)=3 \lambda C_{00}-3 C \lambda^{2} \ln \lambda+0\left(\lambda^{2}\right) .
$$


Indeed, by suitably choosing the terms $0\left(\lambda^{2}\right)$ one gets either the one or two phase region in the limit where $\lambda$ (i.e. $\delta$ ) goes to zero. Therefore, the field theory methods, if they were applied to the lattice uniformly in the lattice spacing $\delta$, would give the first two corrections to the mean field critical point in three dimensions. However, our argument gives the first correction in any dimension and, if it could be refined, would give a new proof of the existence of a mass gap for weakly coupled $\lambda: \phi^{4}:_{3}$. This was recently done for $\lambda: \phi^{4}:_{2}$ by somewhat similar arguments [40].

2) If we use the field theory picture in two dimensions we start with

$$
\begin{aligned}
H= & 1 / 2 \sum_{x, y}\left(\phi_{x}-\phi_{y}\right)^{2}+\lambda_{0} / 4 \sum_{x} \phi_{x}^{4}-3 / 2 C_{00}^{m, \delta} \lambda_{0} \delta^{2} \sum_{x} \phi_{x}^{2} \\
& +\left(m^{2}-\alpha^{2}\right) / 2 \delta^{2} \sum_{x} \phi_{x}^{2} .
\end{aligned}
$$

In two dimensions, $C_{00}^{m, \delta}=(2 \pi)^{-1} \ln \delta+0(1)$. Again we define $\lambda_{0} \delta^{2}=\lambda$,

$$
\left.\sigma=3 / 4 \pi \lambda \ln \lambda+\lambda(-3 / 4 \pi) \ln \lambda_{0}+\left(m^{2}-\alpha^{2}\right) / \lambda_{0}\right]
$$

and as in 1) the field theory result would give

$$
\sigma_{c}(\lambda)=3 / 4 \pi \lambda \ln \lambda+o(\lambda \ln \lambda) .
$$

This formula is actually proven by combining the results of [40] and [44].

If we translate this result for the Ising model by approximating it by a $\phi^{4}$ theory with the identification (13), we get

$$
\beta(\gamma) \simeq 1+\gamma^{2} / \pi \log \gamma .
$$

However, Fisher's mean field bounds give that if $\beta<1+\gamma^{2} C_{00}^{\gamma}$ we are in the onephase region. That is, $\beta_{c}^{\text {Fisher }}(\gamma) \simeq 1+\gamma^{2} / 2 \pi \log \gamma$. So, if we believe in the Wickordering picture above, Fisher's bound gives a first order correction which is too small by a factor of 2 in two dimensions.

3. Using Theorem 3, we can derive a rigorous version of the Ginsburg-Landau theory of critical phenomena which seems more or less natural (see e.g. [41] for a review of that theory). We interpret it as a model which considers the value $\lambda=\sigma=0$ in the $\lambda \phi^{4}-\sigma \phi^{2}$ as the critical point of that theory.

Let us approach that point along any line $\sigma=c \lambda$ with $c<3 C_{00}$. This corresponds to the temperature approaching the critical one from above and it is usually assumed that $\sigma$ is proportional to $\left(T-T_{c}\right)$ (and, of course, $\lambda$ has also to be proportional to $\left(T-T_{c}\right)$, otherwise we would not approach any critical point as Theorem 2 shows). Then one can prove that the inverse correlation length $\xi=m^{-1}$ diverges like $\left(T-T_{c}\right)^{-1 / 2}$ and that the susceptibility diverges like $\left(T-T_{c}\right)^{-1}$. What may be more interesting is that the specific heat, defined as

$$
\sum_{x \in \mathbb{Z}^{3}}\left\langle\phi_{0}^{2} \phi_{x}^{2}\right\rangle-\left\langle\phi_{0}^{2}\right\rangle\left\langle\phi_{x}^{2}\right\rangle,
$$

diverges in that limit (like $\left.T-T_{c}\right)^{-1 / 2}$ ). This is different from the usual mean-field theory. (This is the only difference between Ginsburg-Landau and mean field theories.) 
To prove this, let us first restrict the sum $\sum_{x \in \mathbb{Z}^{3}}$ to $|x| \leqq \xi^{1+\varepsilon}$ for some $\varepsilon>0$; then the remainder is clearly bounded as $\lambda \rightarrow 0$. Now for each $x$ in the sum we can do a perturbation expansion with a remainder of order $\lambda^{2}$, so that $\lambda^{2}\left(\xi^{1+\varepsilon}\right)^{3}$ is negligible. It is easy to check that, among all the Gaussian terms the dominant one will be the zeroth order one. But this is just $\sum_{x}\left(C_{0 x}^{m}\right)^{2}$ which diverges like $\xi \simeq\left(T-T_{c}\right)^{-1 / 2}$. The estimate on the divergence of $\xi$ comes from the fact that using Simon's inequality one derives a lower bound on the mass $m \geqq \ln a / r$ where $a / 2 \simeq \sum_{x \in \partial \Lambda}\left\langle\phi_{0} \phi_{x}\right\rangle$ and $r$ is the radius of $\Lambda$. Since our radius can be taken as $\lambda^{-1 / 2-\varepsilon}$ (with $\varepsilon$ as small as one wishes when $\lambda \rightarrow 0$ ) and $a$ can be taken also as small as one wishes,

$$
\lim _{\lambda \rightarrow 0} \frac{\log m(\lambda)}{\log \lambda} \leqq 1 / 2
$$

On the other hand, the expansion for $\left\langle\phi_{0} \phi_{x}\right\rangle$ when $|x| \sim \lambda^{-1 / 2+\varepsilon}$ shows that $\left\langle\phi_{0} \phi_{x}\right\rangle$ is not smaller than $c \lambda^{1 / 2-\varepsilon}$; since

$$
\left\langle\phi_{0} \phi_{x}\right\rangle \leqq\left\langle\phi_{0}^{2}\right\rangle \exp (-m|x|)
$$

holds by reflection positivity (see [42]) this implies

$$
\lim _{\lambda \rightarrow 0} \frac{\log m(\lambda)}{\log \lambda} \geqq 1 / 2 \text {. }
$$

\section{Appendix by E. Speer}

Let $G$ be a connected Feynman graph in $n$ dimensions, with $L$ lines, $V$ vertices, $N$ loops, and superficial divergence $\omega=n N-2 L$; for a subgraph $H$ of $G$ we write $L(H), V(H)$, etc. Each line $l$ of $G$ has cutoff propagator

$$
\Delta_{l}(q)=\left(q^{2}+m_{l}^{2}\right)^{-1} \chi_{\Lambda}\left(q^{2}\right)
$$

where $\Lambda \geqq 1$,

$$
\chi_{\Lambda}\left(q^{2}\right)=\left\{\begin{array}{lll}
1, & \text { if } & q^{2} \leqq \Lambda^{2}, \\
0, & \text { if } & q^{2} \geqq \Lambda^{2}
\end{array},\right.
$$

and either $m_{l}=1$ (massive lines) or $m_{l}=0$ (massless lines). We will assume that $n>2$ and that the massive lines connect all vertices of $G$; these conditions could be replaced by any others guaranteeing the infrared convergence of the cutoff Feynman integral. Our main result is the

Theorem. Let $F_{\Lambda}(p)$ be the (cutoff) Feynman integral for $G$, let $\mu=\sup _{H} \omega(H)$, where $H$ runs over all (not necessarily connected) subgraphs of $G$. Then for some constant $K$ and non-negative integer $v$,

$$
\left|F_{\Lambda}(p)\right| \leqq K \Lambda^{\mu}\left[1+\log ^{v} \Lambda\right] .
$$

(By convention the empty subgraph $H$ has $\omega(H)=0$; hence $\mu \geqq 0$.) 
Proof. $F_{A}$ has the general form $\int d^{n} k_{1} \ldots d^{n} k_{N} \prod_{1}^{L} \Delta_{l}\left(q_{l}^{2}\right)$, where $q_{l}$ is a linear combination of $k_{1}, \ldots, k_{N}$ and the external momenta $p_{i}$. We write

$$
\Delta_{l}\left(q_{l}\right)=\int_{0}^{\infty} \exp \left(-\alpha_{l}^{2}\left(q_{l}^{2}+m_{l}^{2}\right)\right) \chi_{\Lambda}\left(q_{l}^{2}\right) d \alpha_{l}
$$
interchange the $k$ and $\alpha$ integrals, and write $F_{A}=\sum_{\sigma \in S_{L}} F_{A, \sigma}$, where for any
permutation $\sigma \in S_{L}$,

$$
F_{\Lambda, \sigma}(p)=\int_{\alpha_{\sigma(L)} \geqq \ldots \geqq \alpha_{\sigma(1)}} \prod_{1}^{L} d \alpha_{l} \int \prod_{1}^{N} d k_{j} \prod_{1}^{L} \chi_{\Lambda}\left(q_{l}^{2}\right) \exp \left(-\alpha_{l}^{2}\left(q_{l}^{2}+m_{l}^{2}\right)\right) .
$$

We derive the bound (1) for each $F_{\Lambda, \sigma}$ separately and for notational convenience take $\sigma$ to be the identify. Let $l^{\prime}=\max \left\{l \mid m_{l}^{2}=1\right\}$.

Let $G_{0}$ denote the empty subgraph and for $1 \leqq l \leqq L$, let $G_{l}$ be the subgraph formed by lines $1, \ldots, l$ and their vertices; write $N_{l} \equiv N\left(G_{l}\right), \omega_{l} \equiv \omega\left(G_{l}\right)$, etc., and let $l_{1}, \ldots, l_{N}$ satisfy $l_{j}>0, N_{l}=N_{l_{-1}-1}+1$. Then (see e.g. [43]) the $q_{l}$ in (2) may be defined so that $q_{l_{j}}=k_{j}, 1 \leqq j \leqq N$. Using

$$
\begin{aligned}
& \chi_{\Lambda}\left(q_{l}^{2}\right) \exp \left(-\alpha_{l}^{2}\left(q_{l}^{2}+m_{l}^{2}\right)\right) \leqq 1 \text { for } l \neq l_{j}, \\
F_{\Lambda, \sigma}(p) & \leqq \int_{\alpha_{(L)} \geqq \ldots \geqq \alpha_{(1)}} e^{-\alpha_{l^{\prime}}} \prod_{1}^{L} d \alpha_{l} \prod_{j=1}^{N} \int d k_{j} \chi_{\Lambda}\left(k_{j}^{2}\right) \exp \left(-\alpha_{l_{j}} k_{j}^{2}\right) \\
& \leqq C \int_{\alpha_{(L)} \geqq \ldots \geqq \alpha_{(1)}} e^{-\alpha_{l^{\prime}}} \prod_{1}^{L} d \alpha_{l} \prod_{j=1}^{N} \min \left[\Lambda^{n}, \alpha_{l_{j}}^{-n / 2}\right] \\
& =C \int_{\alpha_{(L)} \geqq \ldots \geqq \alpha_{(1)}} e^{-\alpha_{l^{\prime}}} \prod_{1}^{L} g\left(\alpha_{l}\right)^{-n / 2\left(N_{l}-N_{l-1}\right)} d \alpha_{l},
\end{aligned}
$$

where $g(\alpha)=\max \left[\alpha, \Lambda^{-2}\right]$. (Here and in what follows $C$ represents an arbitrary constant.) For $1 \leqq k \leqq L$ we define

$$
E_{\Lambda}^{k}(a)=\int_{a \geqq \alpha_{k} \geqq \ldots \geqq \alpha_{1}} f_{k}(\alpha) \prod_{l=1}^{k} g\left(\alpha_{l}\right)^{-n / 2\left(N_{l}-N_{l}-1\right)} d \alpha_{l},
$$

where $f_{k}=1$ if $k<l^{\prime}, f_{k}=e^{-\alpha_{l^{\prime}}}$ otherwise, so that

$$
F_{\Lambda, \sigma} \leqq C \lim _{a \rightarrow \infty} E_{\Lambda}^{L}(a) \text {. }
$$

Lemma. For $0 \leqq k \leqq L$, let $\pi(k)=\inf \left\{l \leqq k \mid \omega_{l}=\max _{0 \leqq j \leqq k} \omega_{j}\right\}$. Then

$$
E_{\Lambda(a)}^{k} \leqq C_{\Lambda}^{\omega_{\pi(k)}} g(a)^{1 / 2\left[\omega_{\pi(k)}-\omega_{k}\right]}\left[1+\log ^{\nu_{k}}\left(\Lambda^{2}(a)\right)\right],
$$

moreover, for $k \geqq l^{\prime}$ and $a>1$,

$$
E_{\Lambda(a)}^{k} \leqq C_{\Lambda}^{\omega_{\pi(k)}}\left[1+\log ^{\bar{v}_{k}}\left(\Lambda^{2}\right)\right],
$$

for some non-negative integers $v_{k}, \bar{v}_{k}$.

Before proving the lemma we note that from (6) and (4),

$$
F_{\Lambda, \sigma} \leqq C \Lambda^{\omega_{\pi(L)}}\left[1+\log ^{\bar{v}_{L}} \Lambda^{2}\right],
$$

and since $\mu=\max _{\sigma \in S_{L}} \omega_{\pi(L)},(1)$ is established. 
Proof of Lemma. Setting $E_{\Lambda}^{0}(a) \equiv 1$, we have

$$
E_{\Lambda}^{k}(a) \leqq \int_{0}^{a} E^{k-1}(\alpha) g(\alpha)^{-n / 2\left(N_{k}-N_{k}-1\right)} d \alpha
$$

and we proceed inductively. From (5), since $\omega_{k}=\omega_{k-1}+n\left(N_{k}-N_{k-1}\right)-2$,

$$
E_{\Lambda}^{k}(a) \leqq C \Lambda^{\omega_{\pi(k-1)}} \int_{0}^{a} g(\alpha)^{1 / 2\left(\omega_{\pi(k-1)}-\omega_{k}\right)-1}\left[1+\log ^{v_{k-1}}\left[\Lambda^{2} g(\alpha)\right]\right] d \alpha .
$$

The estimate

$$
\int_{0}^{a} g(\alpha)^{p-1}\left[1+\log ^{q}\left[\Lambda^{2} g(\alpha)\right]\right] d \alpha \leqq \begin{cases}C g^{p}(a)\left[1+\log ^{q}\left[\Lambda^{2} g(a)\right]\right], & \text { if } p>0 \\ C\left[1+\log ^{q+1}\left[\Lambda^{2} g(a)\right]\right], & \text { if } p=0 \\ C \Lambda^{-2 p}, & \text { if } p<0\end{cases}
$$

where $q$ is a non-negative integer, will be proved below. Note that $\omega_{\pi(k-1)}-\omega_{k} \geqq 0$ corresponds to $\pi(k)=\pi(k-1)$, while $\omega_{\pi(k-1)}-\omega_{k}<0$ corresponds to $\pi(k)=k$. Thus (7) and (8) yield (5).

To prove (6), we first suppose $k=l^{\prime}$. Then

$$
E_{\Lambda}^{k}(a)=E_{\Lambda}^{k}(1)+\int_{1}^{a} e^{-\alpha} E_{\Lambda}^{k-1}(\alpha) \alpha^{-n / 2\left(N_{k}-N_{k}-1\right)} d \alpha .
$$

Using (5) twice in the right-hand side yields (6):

$$
\begin{aligned}
E_{\Lambda}^{k}(a) \leqq & C \Lambda^{\omega_{\pi(k)}}\left[1+\log ^{\nu_{k}}\left(\Lambda^{2}\right)\right] \\
& +C \Lambda^{\omega_{\pi(k-1)}} \int_{1}^{a} e^{-\alpha} \alpha^{1 / 2\left(\omega_{\pi(k-1)}-\omega_{k}\right)-1}\left[1+\log ^{\nu_{k-1}}\left(\Lambda^{2} \alpha\right)\right] d \alpha \\
\leqq & C \Lambda^{\omega_{\pi(k)}}\left[1+\log ^{\bar{v}_{k}}\left(\Lambda^{2}\right)\right],
\end{aligned}
$$

since $\omega_{\pi(k)} \geqq \omega_{\pi(k-1)}$. If $k>l^{\prime}$, use (5) and (6) inductively:

$$
\begin{aligned}
E_{\Lambda}^{k}(a)= & E_{\Lambda}^{k}(1)+\int_{1}^{a} E^{k-1}(\alpha) \alpha^{-n / 2\left(N_{k}-N_{k}-1\right)} d \alpha \\
\leqq & C \Lambda^{\omega_{\pi(k)}}\left[1+\log ^{\bar{v}_{k}}\left(\Lambda^{2}\right)\right] \\
& +C \Lambda^{\omega_{\pi(k-1)}}\left[1+\log ^{\bar{v}_{k-1}}\left(\Lambda^{2}\right)\right] \int_{1}^{a} \alpha^{-n / 2\left(N_{k}-N_{k-1}\right)} d \alpha .
\end{aligned}
$$

Since the massive lines lie in $G_{k-1}$ and connect all vertices of $G, N_{k}-N_{k-1} \equiv 1$, and since $n>2$, the last integral is bounded. This proves (6).

It remains only to derive the estimate (8) for

$$
I(a) \equiv \int_{0}^{a} g(\alpha)^{p-1}\left[1+\log ^{q}\left[\Lambda^{2} g(\alpha)\right]\right] d \alpha .
$$

For $a \leqq \Lambda^{-2}, g(a)=\Lambda^{-2}$, so that

$$
I(a)=\int_{0}^{a} \Lambda^{-2(p-1)} d \alpha=\Lambda^{-2(p-1)} a \leqq \Lambda^{-2 p},
$$


which yields (8). For $a>\Lambda^{-2}, g(\alpha)=\alpha$; we write

$$
I(a)=\Lambda^{-2 p}+\int_{\Lambda^{-2}}^{a} \alpha^{p-1}\left[1+\log ^{q}\left(\Lambda^{2} \alpha\right)\right] d \alpha
$$

and consider the cases in (8) separately.

(i) $p>0$. Here

$$
\begin{aligned}
I(a) & =\Lambda^{-2 p}+p^{-1}\left[a^{p}-\Lambda^{-2 p}\right]+p^{-1} a^{p} \log ^{q}\left(\Lambda^{2} a\right)-\frac{q}{p} \int_{\Lambda^{-2}}^{a} \alpha^{p-1} \log ^{q-1}\left(\Lambda^{2} \alpha\right) d \alpha \\
& \leqq\left(1+p^{-1}\right) a^{p}\left[1+\log ^{q}\left(\Lambda^{2} a\right)\right] .
\end{aligned}
$$

(ii) $p=0$. Here

$$
\begin{aligned}
I(a) & =1+\log \left(\Lambda^{2} a\right)+(q+1)^{-1} \log ^{(q+1)}\left(\Lambda^{2} a\right) \\
& \leqq 2\left[1+\log ^{(q+1)}\left(\Lambda^{2} a\right)\right] .
\end{aligned}
$$

(iii) $p<0$. Here

$$
\begin{aligned}
I_{a}= & \Lambda^{-2 p}+|p|^{-1}\left[\Lambda^{-2 p}-a^{p}\right]-|p|^{-1} a^{p} \log ^{q}\left(\Lambda^{2} a\right) \\
& +\frac{q}{|p|} \int_{\Lambda^{-2}}^{a} \alpha^{p-1} \log ^{q-1}\left(\Lambda^{2} \alpha\right) d \alpha \\
\leqq & {\left[1+|p|^{-1}+(q /|p|) C\right] \Lambda^{-2 p}, }
\end{aligned}
$$

where we have used (8) inductively to bound the last integral.

Remark. We can obtain a bound on the exponent $v$ appearing in the theorem by tracing the powers of the logarithm through the induction above. We find that we can in fact take $\bar{v}_{k}=v_{k}$ for all $k$ and

$$
v_{k}=\left\{\begin{array}{lll}
0, & \text { if } & \omega_{k}>\omega_{\pi(k-1)}, \\
v_{k-1}+1, & \text { if } & \omega_{k}=\omega_{\pi(k-1)}, \\
v_{k-1}, & \text { if } & \omega_{k}<\omega_{\pi(k-1)} .
\end{array}\right.
$$

Hence we may choose $v$ so that $v+1$ is the length of the longest chain of subgraphs

$$
H_{v} \supsetneqq H_{v-1} \supsetneqq \ldots \supsetneqq H_{1} \supsetneqq H_{0}
$$

satisfying $\omega\left(H_{j}\right)=\mu, j=0, \ldots, v$. In particular, if all subgraphs $H$ of $G$ containing at least one line satisfy $\omega(H)<0$, then $\mu=v=0$ and the usual power counting theorem is recovered.

Acknowledgements. J. Lebowitz and T. Spencer introduced us to the problems discussed in this paper and made several important suggestions. E. Speer provided us with the result of the appendix. We thank them and also D. Brydges, J. Fröhlich, and C. Thompson for interesting discussions.

\section{References}

1. Fröhlich, J., Simon, B., Spencer, T.: Commun. Math. Phys. 50, 79 (1976)

2. Fisher, M.E.: Phys. Rev. 162, 480 (1967)

3. Private communication

4. Klein, A., Landau, L.J.: Commun. Math. Phys. 43, 143 (1975) 
5. Fröhlich, J., Spencer, T.: J. Stat. Phys. 24, 617 (1981)

6. Simon, B.: Commun. Math. Phys. 77, 111 (1980)

7. Kac, M.: Phys. Fluids 2, 8 (1959)

8. Baker, G.A.: Phys. Rev. 122, 1477 (1961); 126, 2071 (1962)

9. Kac, M., Uhlenbeck, G., Hemmer, P.C.: J. Math. Phys. 4, 216 (1963); U.H.K. ibid, p. 229; H.K.U. ibid 5, 60 (1964)

10. Lebowitz, J.L., Penrose, O.: J. Math. Phys. 7, 8 (1966)

11. Brout, R.: Phys. Rev. 115, 824 (1959); 118, 1009 (1960); 122, 469 (1961)

12. Horwitz, G., Callen, H.B.: Phys. Rev. 124, 1757 (1961)

Englert, F.: Phys. Rev. 129, 567 (1963)

Coopersmith, M., Brout, R.: Phys. Rev. 130, 2539 (1963)

13. Lebowitz, J.L., Stell, G., Baer, S.: J. Math. Phys. 6, 1282 (1965)

Stell, G., Lebowitz, J.L., Baer, S., Theumann, W.: J. Math. Phys. 7, 1532 (1966)

14. Siegert, A.J.F., Vezetti, D.J.: J. Math. Phys. 9, 2173 (1968)

Thompson, C.J., Siegert, A.J.F., Vezetti, D.J.: J. Math. Phys. 11, 1018 (1970)

15. Siegert, A.J.F.: In: Statistical mechanics and field theory. Sen, R.N., Weil, C. (eds.). Jerusalem: Israel University Press, 1972

16. Hemmer, P.C., Lebowitz, J.L.: In: Phase transitions and critical phenomena, Vol. 5B, Domb, C., Green, M.S. (eds.). New York: Academic Press 1976

17. Kac, M., Helfand, E.: J. Math. Phys. 4, 1078 (1963)

18. Kac, M., Thompson, C.J.: J. Math. Phys. 10, 1373 (1969)

19. Stell. G., Theumann, W.K.: Phys. Rev. 186, 581 (1969)

20. Griffiths, R.B.: In: Les Houches lectures, 1970; de Witt, C., Stora, R. (eds.). N.Y.: Gordon and Breach, 1971; and In: Phase transitions and critical phenomena, Vol. 1; Domb, C., Green, M.S. (eds.). New York: Academic Press 1972

21. Simon, B.: Ann. Math. 101, 260 (1975)

Guerra, F., Rosen, L., Simon, B.: Commun. Math. Phys. 41, 19 (1975)

22. Nelson, E. : In : Constructive quantum field theory. In : Lecture Notes in Physics, Vol. 25. Velo, G., Wightman, A.S. (eds.). Berlin, Heidelberg, New York: Springer 1973

23. Ruelle, D.: Commun. Math. Phys. 50, 189 (1976). For a similar result in field theory, see, Glimm, J., Jaffe, A.: J. Math. Phys. 13, 1568 (1972)

24. Glimm, J., Jaffe, A., Spencer, T.: Commun. Math. Phys. 45, 203 (1975)

25. Ellis, R., Monroe, J.L., Newman, C.: Commun. Math. Phys. 46, 167 (1976)

26. Bricmont, J.: J. Stat. Phys. 17, 289 (1977)

27. Sokal, A.D.: Princeton University Thesis. Ann. Inst. H. Poincaré (to appear)

28. Bricmont, J., Fontaine, J.-R., Lebowitz, J.L., Spencer, T.: Commun. Math. Phys. 78, 363 (1981)

29. Griffiths, R.B., Hurst, C., Sherman, S.: Math. Phys. 11, 790 (1970)

30. Newman, C.: Commun. Math. Phys. 41, 1 (1975) and J. Math. Phys. 16, 1956 (1975)

31. Private communication

32. Dreissler, W., Landau, L., Perez, J.: J. Stat. Phys. 20, 123 (1979)

33. Griffiths, R.B.: Commun. Math. Phys. 6, 121 (1967)

34. Fröhlich, J., Israel, R., Lieb, E., Simon, B.: Commun. Math. Phys. 62, 1 (1978)

35. Fröhlich, J., Israel, R., Lieb, E., Simon, B. : J. Stat. Phys. 22, 297 (1980)

36. Hegerfeldt, G.C., Nappi, C.: Commun. Math. Phys. 53, 1 (1977)

37. Newman, C.N.: Commun. Math. Phys. 41, 1 (1975); J. Math. Phys. 16, 1956 (1975)

38. Feldman, J., Osterwalder, K.: Ann. Phys. 97, 80 (1976)

39. Magnen, J., Sénéor, R.: Ann. Inst. Henri Poincaré 24, 95 (1976)

40. Brydges, D., Fröhlich, J., Spencer, T.: Commun. Math. Phys. 83, 123 (1982)

41. Ma, S.K.: Modern theory of critical phenomena. Reading, M.A.: Benjamin 1976

42. Schor, R.: Commun. Math. Phys. 53, 213 (1978)

43. Nakanishi, N.: Graph theory and Feynman integrals. New York: Gordon and Breach 1971

44. Bricmont, J., Fontaine, J.-R.: Infrared bounds and the Peierls argument in two dimensions. Preprint

Communicated by E. Lieb

Received June 30, 1981; in revised form January 6, 1982 